ИЗВЕСТИЯ АҚАДЕМИИ НАУК ЭСТОНСКОИ ССР. ФИЗИКА * МАТЕМАТИКА

PROCEEDINGS OF THE ACADEMY OF SCIENCES OF THE ESTONIAN SSR. PHYSICS * MATHEMATICS

$1985,34,2$

удК $62-501.12$

Моника ОИТ

\title{
ВЛИЯНИЕ ПЕРИОДА ДИСКРЕТНОСТИ НА СВОЙСТВА СВЯЗАННОСТИ МНОГОМЕРНЫХ ЛИНЕИНЫХ СИСТЕМ С ДИСКРЕТНЫМ ВРЕМЕНЕМ
}

\author{
(Представил Н. Алумяэ)
}

Выбор периода дискретности при дискретном по времени управлении является проблемой, которая до сих пор не имеет единого общепризнанного решения. Опираясь на теорию передачи информации, определяется верхнее допустимое значение периода дискретности $\left[{ }^{1}\right]$, но подходящий период часто выбирается путем математического моделирования поведения объекта $\left[{ }^{2}\right]$. В последнее время исследуются зависимости свойства управляемости от выбора периода дискретности $\left[{ }^{3}\right]$. В данной работе изучается влияние значения периода дискретности на свойства связанности многомерных объектов.

\section{Исследование связанности многомерных дискретных объектов}

Математическое описание многомерного объекта в пространственном состоянии задано в виде

$$
\begin{aligned}
& \dot{x}(\tau)=A x(\tau)+B u(\tau) \\
& y(\tau)=C x(\tau),
\end{aligned}
$$

где $x(\tau)-n$-мерный вектор состояний, $u(\tau)-m$-мерный вектор входов, $y(\tau)-p$-мерный вектор выходов, $A, B, C$ - матрицы размерностью $n \times n, n \times m$ и $p \times n$ соответственно. При дискретном управлении объект соединен с цифро-аналоговыми (ЦА) и аналого-цифровыми преобразователями (АЦ). Если АЦ-преобразователем является простой импульсный элемент и ЦА-преобразователем - фиксатор нулевого порядка, то, предполагая, что моменты квантования преобразователей совпадут и период дискретности постоянный, получим уравнения дискретной системы

$$
\begin{aligned}
x[(t+1) \Delta] & =F x(t \Delta)+G u(t \Delta), \\
y(t \Delta) & =H x(t \Delta),
\end{aligned}
$$

где матрицы $F, G, H$ выражаются [ $\left.{ }^{4}\right]$

$$
\begin{aligned}
& F=\exp (A \Delta), \\
& G=\left[\int_{0}^{\Delta} \exp (A \eta) d \eta\right] \cdot B, \\
& H=C .
\end{aligned}
$$

Связанными считают объекты в случае, если все входные переменные влияют более или менее равномерно на все выходы. По уравнениям (2), полученным в результате теоретического исследования пове- 
дения системы или при помощи идентификаций, трудно судить о связа丿нности системы. Для определения связанности отдельных вход-выход пар по описанию в пространстве состоянии используют т. н. матрицы связи $\left[{ }^{5}\right]$, элементами которых являются пределы переходных функций соответствующих входов и выходов, или, в принципе, такого-же типа нормированные матрицы связи $\left[{ }^{6}\right]$. Рассматриваемые в данной работе относительные переходные функции [ $\left.{ }^{7}\right]$ и относительные частотные характеристики $\left[{ }^{8}\right]$ отличаются от предыдущих свободностью от единиц измерения, что позволяет сравнить свойства связанности разных входвыход пар, а также разных систем.

\section{Определение относительной переходной функции}

Для исследования связанности пары $\left(y_{i}, u_{l}\right)$, состоящей из $l$-го входа и $i$-го выхода, перепишем уравнения (2) в виде

$$
\begin{aligned}
x[(t+1) \Delta] & =F x(t \Delta)+g_{l} u_{l}(t \Delta)+\bar{G}_{i} \bar{u}_{l}(t \Delta), \\
y_{i}(t \Delta) & =h^{\prime}{ }_{i} x(t \Delta), \\
\bar{y}_{i}(t \Delta) & =\bar{H}_{i} x(t \Delta),
\end{aligned}
$$

где $u_{l}(t \Delta)-l$-й элемент вектора входов $u, \bar{u}_{l}(t \Delta)-$ вектор входов $u$ без $l$-го элемента, $y_{i}(t \Delta)-i$-й элемент вектора выходов $y, \bar{y}_{i}(t \Delta)-$ вектор выходов $y$ без $i$-го элемента, $g_{l}-l$-й столбец матрицы $G, \bar{G}_{l}-$ матрица $G$ без $l$-го столбца, $h_{i}-i$-й ряд матрицы $H, \bar{H}_{i}-$ матрица $H$ без $i$-го ряда.

Переходная функция представляет собой реакцию выхода на единичный скачок во входе. Обозначим через $p_{i l}(t \Delta)$ переходную функцию пары $\left(y_{i}, u_{l}\right) . p_{i l}(t \Delta)$ определяется как реакция $i$-го выхода на единичный скачок в $l$-м входе, остальные входы нулевые

$$
p_{i l}(t \Delta)=\left[\left.y_{i}(t \Delta)\right|_{u_{l}(t \Delta)=1, \bar{u}_{l}(t \Delta)=0, x(0)=0}\right] .
$$

Если вход $u_{l}(t \Delta)$ не имеет влияния на остальные выходы $\bar{y}_{i}(t \Delta)$, можно сказать, что вход $u_{l}(t \Delta)$ связан только с выходом $y_{i}(t \Delta)$. Для исследования влияния $u_{l}(t \Delta)$ на выходы $\bar{y}_{i}(t \Delta)$ требуем, чтобы все $\bar{y}_{i}(t \Delta)=0$, и рассмотрим соответствующую переходную функцию $q_{i l}(t \Delta)$

$$
q_{i l}(t \Delta)=\left[\left.y_{i}(t \Delta)\right|_{u_{l}(t \Delta)=1, \bar{y}_{t}(t \Delta)=0, x(0)=0}\right] .
$$

В таком случае учитываем также влияние $\bar{u}_{l}(t \Delta)$ на $y_{i}(t \Delta)$. Если числа входов и выходов объекта равны $m=p$, то регулятором, обеспечивающим нулевой выход при любых входных воздействиях, является обратная динамическая система системы (4) из [ $\left.{ }^{9}\right]$. Если переходные функции (5) и (6) существенно не различаются, то это значит, что вход $u_{l}(t \Delta)$ имеет в основном влияние на выход $y_{i}(t \Delta)$. Относительная переходная функция $\varphi_{i l}(t \Delta)$ определена как отношение переходных функций $p_{i l}(t \Delta)$ и $q_{i l}(t \Delta)$

$$
\varphi_{i l}(t \Delta)=p_{i l}(t \Delta) / q_{i l}(t \Delta) .
$$

Если относительная переходная функция мало отличается от единицы, пара $\left(y_{i}, u_{l}\right)$ является слабосвязанной. Признаком автономности является $\varphi_{i l}(t \Delta)=1$. По параметрам исходной системы (4) относительная переходная функция $\varphi_{i l}(t \Delta)$ выражается $\left[{ }^{8}\right]$

$$
\varphi_{i l}(t \Delta)=\sum_{k=1}^{t} h^{\prime}{ }_{i} F^{k-1} g_{l}\left[\sum_{k=1}^{t} h^{\prime}{ }_{i}\left(M_{i l} F\right)^{k-1} M_{i l} g_{l}\right]^{-1},
$$

где $M_{i l}=I-\bar{G}_{l}\left(\bar{H}_{i} \bar{G}_{l}\right)^{-1} \bar{H}_{i}$. 


\section{Зависимостть относительной переходной функции от выбора периода дискретности}

Так как значения всех параметров дискретного объекта зависят от выбора периода дискретности $\Delta$, то относительные переходные функции являются сложными неявными функциями от $\Delta$. Выразить эту зависимость аналитически практически невозможно. Поэтому приходится опираться на экспериментальные исследования. С помощью системы машинного проектирования $\left[{ }^{10}\right]$ изучены свойства связанности при различных периодах дискретности ряда показательных объектов. Оказалось, что относительные переходные функции $\varphi_{i l}(t \Delta)$ практически независимы от значения периода дискретности.

\section{Относительные частотные характеристики}

Для исследования связанности в частотной области преобразуем системы (4) $z$-преобразованием

$$
\begin{aligned}
z X(z)-z X(0) & =F X(z)+g_{l} U_{l}(z)+\bar{G}_{l} \bar{U}_{l}(z), \\
Y_{i}(z) & =h^{\prime}{ }_{i} X(z), \\
\bar{Y}_{i}(z) & =\bar{H}_{i} X(z),
\end{aligned}
$$

где $z-$ комплексная переменная $z=e^{j \omega \Delta t}, \omega-$ приведенная частота, $\Delta$ - период дискретности, $X(z), U(z), Y(z)$ - z-преобразования переменных $x(t \Delta), u(t \Delta)$ и $y(t \Delta), t=0,1,2, \ldots$ соответственно. Рассмотрим вход-выход пары $\left(Y_{i}, U_{l}\right)$. Обозначим через $P_{i l}(j \omega \Delta t)$ частотную характеристику пары $\left(Y_{i}, U_{l}\right)$, которая определяется как реакция $i$-го выхода на входную переменную $U_{l}=\exp (j \omega \Delta t) \quad\left[{ }^{4}\right]$, остальные входы нулевые

$$
P_{i l}(j \omega \Delta t)=\left[\left.Y_{i}(j \omega \Delta t)\right|_{U_{l}(j \omega \Delta t)=\exp (j \omega \Delta t), \bar{U}_{l}(j \omega \Delta t)=0, X(0)=0}\right] .
$$

Для определения влияния $U_{l}(j \omega \Delta t)$ на остальные выходы $\bar{Y}_{i}(j \omega \Delta t)$ регулируем объект обратной системой $\left[{ }^{9}\right]$ так, что все $\bar{Y}_{i}(j \omega \Delta t)=0$. Соответствующая частотная характеристика $Q_{i l}(j \omega \Delta t)$ пары $\left(Y_{i}, U_{l}\right)$ определяется

$$
Q_{i l}(j \omega \Delta t)=\left[\left.Y_{i}(j \omega \Delta t)\right|_{U_{l}(j \omega \Delta t)=\exp (j \omega \Delta t), \bar{Y}_{l}(j \omega \Delta t)=0, X(0)=0}\right] .
$$

Если частотные характеристики (10) и (11) существенно не различаются, то это значит, что вход $U_{l}(j \omega \Delta t)$ имеет влияние в основном на выход $Y_{i}\left(j_{\omega} \Delta t\right)$. В таком случае вход-выход пара автономная. Соответствующая относительная частотная характеристика $\psi_{i l}(j \omega \Delta t)$ определена как отношение $P_{i l}(j \omega \Delta t)$ и $Q_{i l}(j \omega \Delta t)$

$$
\psi_{i l}(j \omega \Delta t)=P_{i l}(j \omega \Delta t) / Q_{i l}(j \omega \Delta t) .
$$

Для определения относительных амплитудных и фазовых характеристик используем вид

$$
\begin{aligned}
& P_{i l}(j \omega \Delta t)=P_{i l}(\omega \Delta t) \exp \left[j \Phi_{i l}^{P}(\omega \Delta t)\right], \\
& Q_{i l}(j \omega \Delta t)=Q_{i l}(\omega \Delta t) \exp \left[j \Phi_{i l}^{Q}(\omega \Delta t)\right], \\
& \psi_{i l}(j \omega \Delta t)=\psi_{i l}(\omega \Delta t) \exp \left[j \Phi_{i l}^{\Psi}(\omega \Delta t)\right],
\end{aligned}
$$

где $\psi_{i l}(\omega \Delta t)$ и $\Phi_{i l}(\omega \Delta t)$ выражаются 


$$
\begin{aligned}
\psi_{i l}(\omega \Delta t) & =P_{i l}(\omega \Delta t) / Q_{i l}(\omega \Delta t), \\
\Phi_{i l}^{\psi}(\omega \Delta t) & =\Phi_{i l}^{P}(\omega \Delta t)-\Phi_{i l}^{Q}(\omega \Delta t),
\end{aligned}
$$

где $\psi_{i l}(\omega \Delta t)$ - относительная амплитудная частотная характеристика, $\Phi_{i l}{ }^{\psi}(\omega \Delta t)$ - относительная фазовая частотная характеристика.

При несвязанных вход-выход парах частотные характеристики $P_{i l}(j \omega \Delta t)$ и $Q_{i l}(j \omega \Delta t)$ равны и относительные частотные характеристики в таком случае

$$
\begin{aligned}
& \psi_{i l}(\omega \Delta t)=P_{i l}(\omega \Delta t) / Q_{i l}(\omega \Delta t)=1, \\
& \Phi_{i l}^{\psi}(\omega \Delta t)=\Phi_{i l}^{P}(\omega \Delta t)-\Phi_{i l}^{Q}(\omega \Delta t)=0 .
\end{aligned}
$$

Отсюда вытекают условия декомпозиции в частотной области: входвыход пара является автономной в области частот, где относительная амплитудная характеристика $\psi_{i l}(\omega \Delta t)=1$ и относительная фазовая характеристика $\Phi_{i l}{ }^{\psi}(\omega \Delta t)=0$.

\section{Зависимость относительных частотных характеристик от выбора периода дискретности}

Относительные частотные характеристики $\psi_{i l}(\omega \Delta t)$ и $\Phi_{i l} \psi^{\psi}(\omega \Delta t)$ (14) являются функциями частоты $\omega$ и периода дискретности $\Delta$. Выразить аналитическую зависимость относительных частотных характеристик (14) от периода дискретности практически невозможно; так как все составляющие формулы (14) сами являются сложными функциями от $\Delta$. В аргументе тригонометрических функций период дискретности опреде-
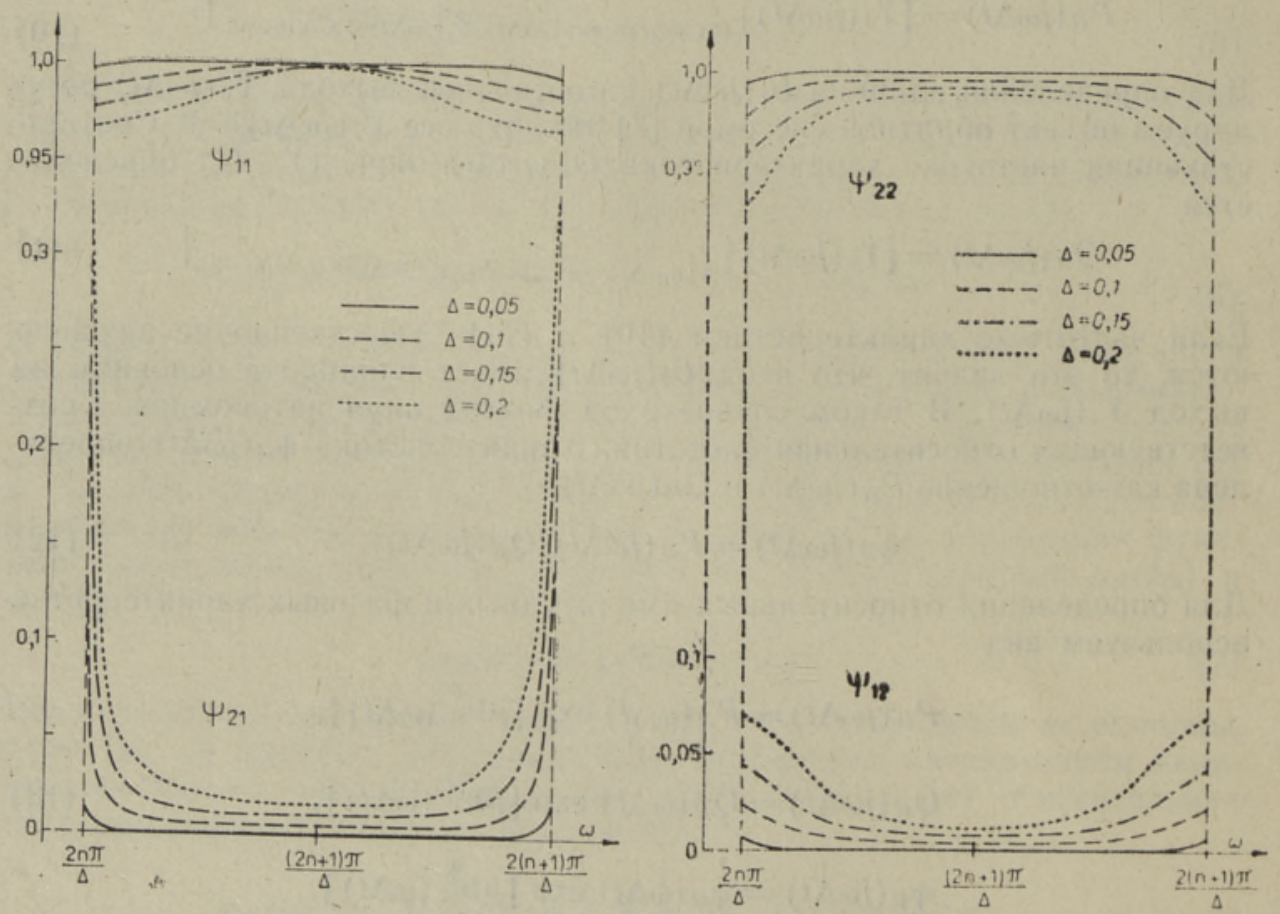

Рис. 1. Зависимость амплитудных частотных характеристик от периода дискретности (в относительном масштабе). 

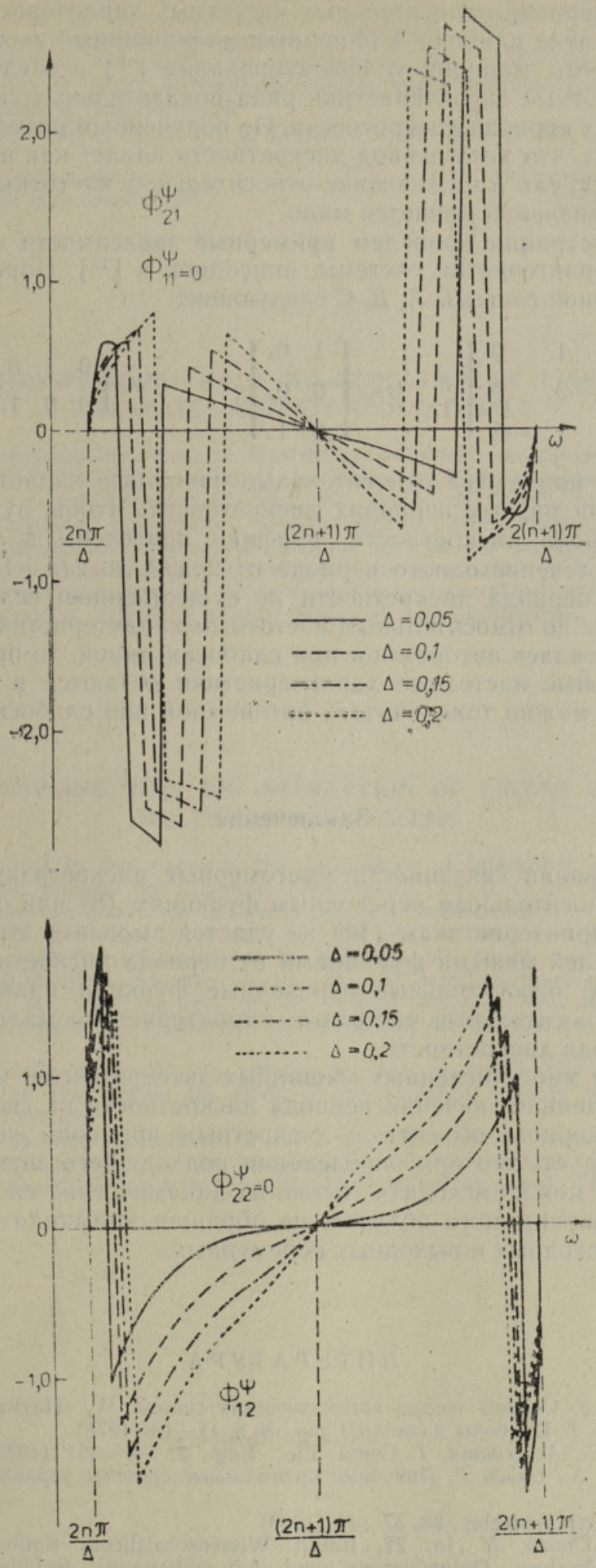

Рис. 2. Зависимость относительных фазовых частотных характеристик от периода дискретности (в относительном масщтабе). 
ляет длину периода относительных частотных характеристик. Дальнейшее исследование влияния $\Delta$ опирается на машинный эксперимент. При помощи системы машинного проектирования $\left[{ }^{10}\right]$ исследованы относительные частотные характеристики ряда показательных систем при разных значениях периода дискретности. По полученным результатам можно сделать вывод, что хотя период дискретности влияет как на определение периодичности, так и на значение относительных частотных характеристик, область значений меняется мало.

Для иллюстрации приведем примерные зависимости относительных частотных характеристик системы, описанной в [ $\left.{ }^{11}\right]$. Параметры исходной непрерывной системы $A, B, C$ следующие:

$$
A=\left[\begin{array}{rrr}
-3 . & 1 . & 0 . \\
2 . & -3 . & 2 . \\
0 . & 1 . & -3 .
\end{array}\right], \quad B=\left[\begin{array}{ll}
1 . & 0 . \\
0 . & 0 . \\
0 . & 1 .
\end{array}\right], \quad C=\left[\begin{array}{lll}
0 . & 1 . & 0 \\
0 . & 0 . & 1 .
\end{array}\right]
$$

На рис. 1, 2 приведены относительные частотные характеристики системы (16) при разных периодах дискретности. Чтобы лучше выделить влияние периода дискретности, графики приведены в относительном масштабе (в течение одного периода от $2 n \pi / \Delta$ до $2(n+1) \pi / \Delta)$. Видно, что влияние периода дискретности не существенное: если при какомнибудь $\Delta$, судя по относительным частотным характеристикам, вход-выход пара оказалась автономной или слабосвязанной, то при изменениях $\Delta$ относительные частотные характеристики остаются в пределах, где данную пару можно тоже считать автономной или слабосвязанной.

\section{Заключение}

При исследовании связанности многомерных дискретизированных систем по их относительным переходным функциям (8) или относительным частотным характеристикам (14) не удается выразить этих относительных показателей явными функциями от периода дискретности. Удалось выяснить, что относительные переходные функции практически независимы, а относительные частотные характеристики мало зависимы от выбора периода дискретности.

На основе многочисленных машинных экспериментов можно сделать вывод, что влияние значения периода дискретности на свойство связанности многомерного объекта с дискретным временем незначительное. Из этого следует, что при определении подходящего значения периода дискретности можно исходить только из динамических свойств рассматриваемого непрерывного объекта, не обращая внимания на взаимосвязанность его входных и выходных переменных.

\section{Л И ТЕ Р А Т Р А}

1. Цыпкин Я. З. Основы теории автоматических систем. М., «Наука», 1977.

2. Филиппов Л. Г. Приборы и системы упр., №.8, 11-13 (1978).

3. Hirzinger, G., Ackermann, J. Comp. Elec. Eng., 2, 347-351 (1975).

4. Квакернаак X., Сиван P. Линейные оптимальные системы управления. М., «Мир», 1977.

5. Söte, W. Regelungstechn., 28, 37-44 (1980).

6. Raske, F., Thoma, M. In: 27. Intern. Wissenschaftliches Kolloquium, Vortragsreihe *Analyse, Optimisierung und Automatisierung technischer und nichttechnischer Prozesse». Ilmenau, DDR, 1982, 3-7.

7. Jaaksoo, 0 . In: The 2nd IFAC/IFIP Symposium Software for Computer Control. Prague, 2, A-XXV, 1979. 
8. Яаксоо Ю. И., Ойт М. Э. В кн.: Тез. докл. III Всесоюзного совещания по автоматизашии проектировання системы автоматического и автоматизированного управления технологическими процессами. М., 1981, 161-162.

9. Яаксоо Ю. И. Изв. АН СССР. Техн. кибернетика, № 5, 165-169 (1980).

10. Ойт М., Яаксоо Ю. В кн.: Автоматизированное проектирование систем управления. M., «Наука», 4, 1982, 145-155.

11. Alevisakis, G., Seborg, D. E. Int. J. Contr., 17, 541-551 (1973).
Институт кибернетики
Академии наук Эстонской ССР
Поступнла в редакцию
1/XI 1983

Monika OIT

\section{KVANTIMISPERIOODI MOJU MITMEMOOTMELISE LINEAARSE DISKREETSE OBJEKTI SEOSTATUSELE}

On uuritud mitmemõõtmelise lineaarse ajas diskreetse objekti seostatuse sõltuvust kvantimisperioodi valikust. Objekti seostatust on iseloomustatud suhteliste siirdefunktsioonide ja suhteliste sageduskarakteristikutega, mis avalduvad objekti parameetrite kaudu keerukate matemaatiliste avaldistena. Otsest sõltuvust kvantimisperioodist on praktiliseit võimatu analüütiliselt väljendada. Seetõttu tugineb töö peamiselt juhtimissüsteemide masinprojekteerimissüsteemi abil tehtud eksperimentaalsel uurimisel. Osutub, et nii suhtelised siirdefunktsioonid kui ka suhtelised sageduskarakteristikud on vähe sôltuvad kvantimisperioodist. Järelikult objekti seostatust kvantimisperioodi valikul arvestada ei tule.

\section{Monika OIT}

\section{EFFECT OF SAMPLING TIME ON INTERACTION OF LINEAR DISCRETE TIME MULTIVARIABLE SYSTEMS}

The problem treated in this paper is the dependence of interaction of linear discrete time multivariable systems on sampling time. Interaction is characterized by relative transient response functions and relative frequency characteristics.

Let $u_{l}$ denote the $l$-the input and $y_{i}$ - the $i$-th output of multivariable control system (2). To study the interaction of the pair $\left(y_{i}, u_{l}\right)$, let us give the system form (4). The relative transient response furiction $\varphi_{i l}(8)$ describes the interaction of inputoutput pair $\left(y_{i}, u_{l}\right)$ with the rest of the system. Relative frequency characteristics $\psi_{i i}(\omega \Delta t)$ and $\Phi_{i l} \psi(\omega \Delta t)(14)$ describe the interaction of the pair $\left(y_{i}, u_{l}\right)$ in frequency domain.

It is practically impossible to express $\varphi_{i l}(t \Delta), \psi_{i l}(\omega \Delta t)$ and $\Phi_{i l} \psi(\omega \Delta t)$ as a function of $\Delta$. For that reason the effect of the sampling time is studied experimentally, using CAD-system.

It turned out that dependence of both relative transient response function and relative frequency characteristics on sampling time is insignificant. Accordingly, interaction can be neglected when solving the problem of the best choice of sampling time. 